\title{
Revisiones
}

\section{Tuberculosis en nuevos escenarios: establecimientos penitenciarios}

\author{
Eduardo Zarate ${ }^{1}$, Irma Lobón ${ }^{2}$, Carlos Saavedra ${ }^{3}$, María Castañeda ${ }^{4}$ \\ “...acá la gente se enferma de muchas cosas. Se enferman de SIDA, de tuberculosis... \\ pero muchas veces no nos hacen caso. Se enferman, porque muchas veces hacemos \\ cosas malas...(Carlos, interno con TBC en penal Lurigancho...” (Portocarrero, 2004)
}

\begin{abstract}
Resumen Los autores revisan la incidencia de tuberculosis (TBC) y virus de inmunodeficiencia humana (VIH) en las personas privadas de su libertad. En los principales establecimientos penitenciarios del Perú, estas incidencias son más altas que en la población general. Se revisa las condiciones que contribuyen dichas tasas y se propone una estrategia diferenciada para dichas poblaciones de riesgo: una intervención de capacitación en los profesionales de la salud, formación de promotores de salud y un intenso uso de la información, educación y comunicación en salud.
\end{abstract}

Palabras clave Tuberculosis; HIV; Perú, tuberculosis; centro de rehabilitación.

Tuberculosis new scenery: prison establishments Abstract

This is a review on the incidence of tuberculosis (TBC) y human immunodeficiency virus (HIV) in imprisoned persons. These incidences are higher in the main Peruvian penitentiary establishments than in the general population. We review the contributing conditions and a differentiated strategy for those populations at risk is proposed: a capacitating intervention of health professionals, health promoters formation, and intense use of health information, education and communication.

1 Profesor del Departamento de Medicina Preventiva y Salud Pública. Facultad de Medicina, UNMSM. Lima, Perú.

2 Profesora del Departamento de Ciencias Dinámicas. Facultad de Medicina, UNMSM. Lima, Perú.

${ }^{3}$ Profesor del Departamento de Medicina. Facultad de Medicina, UNMSM. Lima, Perú.

4 Profesora del Departamento de Medicina. Facultad de Medicina, UNMSM. Lima, Perú.
Keywords: Tuberculosis; HIV; Peru, tuberculosis; rehabilitation center.

\section{INTRODUCCIÓN}

La salud en las prisiones es a menudo olvidada o considerada de baja prioridad. Los presos son excomulgados y no escuchados. La sociedad ve con ambivalencia una atención sanitaria de calidad a las personas acusadas de actuar contra la sociedad. La salud de una persona privada de su libertad (PPL) es responsabilidad de diferentes autoridades mal coordinadas entre sí. El clima de desorganización y violencia agrava la situación, obstaculiza el acceso a la atención médica y fomenta comportamientos perturbados.

Los internos son encarcelados como castigo, no para castigarlos $\left({ }^{1}\right)$. Esta frase significa que la pérdida del derecho a la libertad se hace 
efectiva con la reclusión en un recinto cerrado. No obstante, el perder su libertad no significa la pérdida de otros derechos que mantiene, como el derecho a la salud. El hecho de mantener a una persona bajo custodia del Estado no debería ser perjudicial para su salud. Desgraciadamente, esto es precisamente lo que sucede en muchos establecimientos penitenciarios.

En cualquier país con escasos recursos, las personas privadas de su libertad suelen ser la última prioridad presupuestaria. Son los extremos de los marginados. Viven con frecuencia hacinadas en instalaciones con insuficiente ventilación, higiene y saneamiento. La comida institucional suele ser escasa y poco nutritiva. Los comportamientos ilegales, como el consumo de alcohol y drogas, las relaciones sexuales (consentidas o no) pueden tener lugar sin control. Estas condiciones son un buen caldo de cultivo para las enfermedades infecciosas crónicas, incluyendo la tuberculosis (TBC) y el virus de la inmunodeficiencia adquirida (VIH).

Los problemas de salud en las prisiones son el resultado de una compleja interacción entre la pobreza, el encarcelamiento y las enfermedades. Deben de llevarse a cabo urgentes intervenciones, como el acceso universal a la estrategia de terapia directamente observada (DOTS) y la búsqueda activa de sintomáticos respiratorios. Pero, estas acciones deben estar incluidas en un contexto más amplio contra los factores que facilitan y perpetúan la enfermedad en las prisiones. Entre ellas, la reducción del hacinamiento, la promoción y ejercicio de los derechos en salud, la coordinación del sistema de salud de las prisiones integradas con las estrategias sanitarias nacionales y un adecuado sistema de referencia y contrarreferencia. Para el logro de estas metas, se hace necesario el trabajo intersectorial.

Controlar las enfermedades crónicas, como la TBC, el VIH/SIDA y la coinfección TBC-VIH/ SIDA, en lugares tan difíciles como las prisiones, donde confluyen aspectos sociales, culturales y psicológicos por parte de las personas privadas de su libertad, y aspectos normativos, de seguridad, falta de presupuesto, limitaciones técnicas sanitarias por parte de la Institución penitenciaria, requiere de un amplio conjunto de intervenciones orientadas hacia aspectos sanitarios y otras más hacia aspectos administrativos y de ordenamiento institucional. Por otro lado, el control de estas enfermedades en las prisiones no debe ser visto como un programa técnico aislado, sino que debe formar parte de un esfuerzo amplio e integrado para mejorar la salud dentro y fuera de las prisiones. Las acciones en la institución penitenciaria deben estar incorporadas dentro de las estrategias sanitarias nacionales.

El Perú es el país con mayor morbilidad e incidencia $\left({ }^{2}\right)$ de TB en América Latina y, de igual manera, es el octavo país del mundo con mayor carga de TB multidrogo resistente (MDR) $\left({ }^{3}\right)$. Cada año, la tasa de TB se incrementa aproximadamente en $3 \%$, al que hay que agregar otro $3 \%$ de carga epidemiológica de casos que se contagian y desarrollan la enfermedad $\left({ }^{4}\right)$. Las personas privadas de su libertad ascienden a 30398 , de las cuales $93 \%$ son hombres y $7 \%$ mujeres $\left({ }^{5}\right)$. El $54 \%$ se concentra en nueve establecimientos penitenciarios (EP), considerados los mas grandes del país: Lurigancho (Lima), Castilla (Piura), Callao, Trujillo, Huanuco, Miguel Castro Castro, Picsi (Chiclayo), Socabaya (Arequipa) e Ica. En éstos se concentra el $70 \%$ de los casos de TBC. A pesar de esta situación, no existe en los EP una estrategia sanitaria de control de TBC lo suficientemente fuerte como para intervenir el problema.

La población de personas privadas de su libertad sigue creciendo, con muchas detenciones cortas. Los números reales de personas que pasan por prisión cada año son potencialmente cuatro a seis veces superiores a las registradas. Estas poblaciones se componen mayormente por hombres entre 18 y 44 años, con una mayor representación de grupos marginados de la sociedad, especialmente drogadictos, enfermos mentales y pobres $\left(^{6}\right)$. Al comparar las tasas de TBC en EP con la población general da como resultado que en los EP existe más riesgo de 
enfermarse que fuera de él. Las causas de los niveles tan altos de TBC en las personas privadas de su libertad son:

- Un número alto de personas privadas de su libertad provienen de sectores de la población ya expuestos a altos riesgo de infección TB y de desarrollar la enfermedad (alcohólicos, drogadictos, vagos, enfermos mentales), quienes a menudo no han tenido acceso a un tratamiento adecuado fuera de la prisión.

- Los EP facilitan la transmisión de la infección TB a través de una prolongada y repetida exposición al $M$. tuberculosis como resultado de la detección tardía de casos, la falta de aislamiento, tratamiento insuficiente, alta tasa de rotación de personas privadas de su libertad por traslados dentro del sistema penitenciario, y las condiciones de hacinamiento.

- Las personas privadas de su libertad también corren el riesgo de una rápida progresión de la enfermedad por una reciente infección o reactivación de una infección latente, debido a la coexistencia de otras patologías, particularmente la infección por el VIH, el uso intravenoso de drogas, mala nutrición, estrés físico y emocional.

Las personas privadas de su libertad gozan de derechos inalienables en virtud de tratados y acuerdos internacionales, entre los cuales tienen derechos a la atención de la salud y, por supuesto, derecho a no contraer una enfermedad en el establecimiento penitenciario. Sus derechos en salud y el acceso a los servicios de salud son parte de un problema que debe ser asumido por la sociedad.

\section{LAS PRISIONES COMO RESERVORIOS DE TBC Y VIH}

La finalidad de las políticas de salud pública es garantizar las mejores condiciones posibles a los miembros de la sociedad, a fin de que cada uno pueda permanecer sano. A menudo, las personas privadas de su libertad quedan excluidas de esta ecuación. Los internos entran y salen de los penales. Son puestos en libertad si se les declara inocentes. Van y vienen de un establecimiento a otro durante las investigaciones y el juicio. Es más, con frecuencia son trasladados, por diversas razones, de un EP a otro. Los internos están en contacto con personas que ingresan y salen de las cárceles, trabajadoras sexuales, familiares, guardias, empleados de los EP y personal de salud. Cuando han cumplido su condena, se declara su amnistía, recobran la libertad; pero, si han contraído una enfermedad crónica, ésta es puesta en circulación en la ciudad, diseminándose en la población en general. Lo cual hace que sea más importante controlar cualquier enfermedad contagiosa en el penal, para evitar su propagación al exterior.

Se estima que, en un día, el número de personas en prisión en el mundo es de 8 a 10 millones, puesto que muchas detenciones son cortas. Por ejemplo, en la Federación Rusa la población de personas privadas de su libertad llegaba a 2 millones en el $2000\left({ }^{1}\right)$; el movimiento se calculaba en 300000 personas al año (Tabla 1). En el Perú, la población de personas privadas de su libertad es estimada en 31000 personas y con una rotación anual de 3000 entre los establecimientos penitenciarios. Los EP son considerados como reservorios de TBC y de TBCMDR. Los datos nacionales confirman que en los EP existe 30 veces más riesgo de contraer TB que fuera de él. En el mundo, la atención médica sanitaria debe ser competencia de los servicios sanitarios del ministerio del que depende la prisión. En nuestro caso, dependen del Instituto Nacional Penitenciario (INPE). Debido a los escasos fondos fiscales destinados a la salud pública en general, sólo ocasionalmente serán prioridad para la administración penitenciaria la salud de las personas privadas de su libertad, la cual se interesa más por la seguridad y disciplina. En el último año, existe un esfuerzo de las autoridades del INPE por mejorar las condiciones de salud de los EP, para lo cual se cuenta con apoyo de fondos provenientes de la cooperación internacional. 
Tuberculosis en nuevos escenarios

Tabla 1. Incidencia de TBC activa en población de personas privadas de libertad.

\begin{tabular}{llll}
\hline \multicolumn{1}{c}{ Área } & Año & \multicolumn{1}{c}{$\begin{array}{c}\text { Casos en prisión } \\
\text { por } 100000\end{array}$} & $\begin{array}{l}\text { Población general } \\
\text { casos por } 100000 \\
\text { (todas las formas) }\end{array}$ \\
\hline Brasil & $1992-3$ & 5,714 (TB pulmonar) & $55,9(1192)$ \\
Georgia & $1997-8$ & 5,995 & $155(1997)$ \\
Malawi & 1996 & 5,142 (TB pulmonar) & $17,7(1197)$ \\
Ruanda & $1996-8$ & 3,363 (Todas las formas) & $209,5(1996)$ \\
España (Madrid) & $1993-4$ & 2,283 (Todas las formas) & $79,3(1997)$ \\
China (Taiwán) (23) & $1997-8$ & 259 (TB pulmonar) & $24(1993)$ \\
EEUU (Nueva York) & 1991 & 156,2 (Todas las formas) & 10,4 \\
Perú & 2003 & 1,725 & 107,7 \\
\hline
\end{tabular}

Fuentes: a.- El control de la TBC en las prisiones. OMS. Comité Internacional de la Cruz Roja, Barcelona, España, 2002 $\left.{ }^{6}{ }^{6}\right)$ b.- Línea de base de los nueve establecimientos principales penales del Perú. Fortalecimiento de la prevención y control del SIDA y la TB en el Perú. Agosto 2004 (7). Elaboración propia.

La TB, en su forma más agresiva, la resistente, puede ser más frecuente en las prisiones, ya que los factores que fomentan la transmisión de la TBC facilitan la propagación de la TB MDR. Además, varios de los aspectos de los EP facilitan el desarrollo de TB-MDR, siendo el principal los escasos recursos fiscales y la pobre atención sanitaria que el Estado le dedica, pudiéndose generar suministros irregulares de medicamentos y tratamientos insuficientes. Resulta preocupante la población de TBC-MDR en los establecimientos penitenciarios; las informaciones internacionales revelan los datos mostrados en la Tabla 2.

Asimismo, se ha demostrado que el VIH se encuentra en las prisiones del mundo, y nuestro país no es la excepción (Tabla 3). Esto debe ser de suma preocupación no sólo para las personas privadas de su libertad y las autoridades de los penales, sino también para el personal que labora en las cárceles, los encargados de la salud pública y la sociedad en su conjunto. La prevalencia de enfermedades de transmisión sexual y el VIH es más alta en las cárceles que en la población general y sigue aumentando, debido a que estos establecimientos son un terreno propicio para su transmisión. Un estudio sobre vigilancia biológica y el comportamiento de la infección del VIH y la
Tabla 2. Tasas declaradas de TB multidrogro resistente (MDR) en personas privadas de su libertad.

\begin{tabular}{llr}
\hline \multicolumn{1}{c}{ País (estudio) } & Año & $\begin{array}{r}\text { Tasa de } \\
\text { TB-MDR }\end{array}$ \\
\hline Azerbaiyán (n=131) & 1997 & $23,0 \%$ \\
Georgia (n=276) & $1997-8$ & $13,0 \%$ \\
Federación Rusa (Mariinsk) $(\mathrm{n}=164)$ & 1998 & $22,6 \%$ \\
España (Madrid) $(\mathrm{n}=203)$ & 1994 & $5,9 \%$ \\
EEUU (Nueva York) $(\mathrm{n}=116)$ & 1991 & $32,0 \%$ \\
\hline
\end{tabular}

Fuente: Informe del Taller Internacional de control de la Tuberculosis en Centros Penitenciarios. Honduras. 2003.

sífilis en 20 establecimientos penitenciarios en el Perú, en enero 2000, demostró una prevalencia de VIH de $1,1 \%$ y la prevalencia de sífilis fue $4,4 \%$ (Tabla 3). Los comportamientos de riesgo más importantes fueron el haber estado recluido previamente en un EP y consumir pasta básica de cocaína $\left({ }^{8}\right)$.

Los EP muestran una alta concentración de factores de riesgo para que se transmita la infección por VIH: 1) Un alto porcentaje de personas privadas de su libertad proceden de ambientes con una alta prevalencia de infección por VIH; 2) Estos grupos tienen comportamientos de riesgo, como el uso de drogas, y las prácticas sexuales inseguras 
Tabla 3. Seroprevalencia VIH observada en personas privadas de su libertad (PPL).

\begin{tabular}{lcc}
\hline \multicolumn{1}{c}{ País } & Año & $\begin{array}{c}\text { Prevalencía } \\
\text { VIH en PPL }\end{array}$ \\
\hline Brasil (Sao Paulo) & 1995 & $14,4 \%$ \\
Etiopia (Dire Dawa) & 1998 & $6,0 \%$ \\
Escocia (Glasgow) & 1994 & $0,9 \%$ \\
EEUU & $1988-1999$ & $4,1 \%$ \\
Perú & 2000 & $1,1 \%$ \\
\hline
\end{tabular}

Elaboración propia. Fuente: Informe del Taller Internacional de control de Tuberculosis en Centros Penitenciarios. Honduras, 2003. J Best y col: El VIH en establecimientos penitenciarios. Edición para América Latina. Organización Mundial de la Salud. Oficina regional para Europa. Comité Internacional de la Cruz Roja, Lima, 2001.

ocurren con frecuencia ${ }^{1}$; 3) No se reconoce oficialmente la existencia de estos comportamientos de riesgo y la circulación de trabajadoras sexuales y el consumo de drogas son prácticas cotidianas en los EP del país ${ }^{2}$; 4) Las intervenciones para reducir el riesgo de infección por VIH, como el suministro de condones, pueden ser consideradas inaceptables por factores culturales de las personas privadas de su libertad; 5) Existe un alto número de tatuajes realizados con equipos no estériles.

Por otro lado, las prisiones están sobrepobladas y dentro de ellas se desarrolla un clima de violencia y miedo. Abundan las tensiones, incluidas las de carácter sexual. Lo retardado y engorroso de los procesos judiciales, mantiene a un considerable número de personas privadas de su libertad sin sentencia judicial ${ }^{3}$. A menudo, los internos encuentran el alivio de esas tensiones y del aburrimiento de la vida penitenciaria en el consumo de drogas y/o en la práctica de relaciones sexuales de riesgo. El VIH exacerba el ya elevado riesgo de TB de los PPL, propiciando la coinfección TBC-VIH.

\section{TBC EN ESTABLECIMIENTOS PENITENCIARIOS DEL PERÚ}

Aún cuando el país ha realizado un gran esfuerzo de control de TBC, reconocido a nivel internacional, reporta $25 \%$ de todos los casos de TBC en Latinoamérica. La incidencia de esta enfermedad ha ido disminuyendo en los últimos 10 años, a raíz de la implementación de la estrategia DOTS, desde 1991. El año 1992, la incidencia de TBC en todas sus formas fue de 243 casos por $100 \mathrm{mil}$; el año 2000, registró 134 casos por $100000 \mathrm{y}$, en el 2003,107,7 casos por $100000\left(^{7}\right)$. La incidencia no es uniforme, concentrándose principalmente en Lima y Callao, es decir que, a mayor concentración de población mayor es el riesgo de transmisión, debido al contacto próximo con pacientes bacteriológicamente positivos. El factor de riesgo fundamental en estos lugares es el hacinamiento, muy común en los asentamientos humanos y en los barrios marginales. A éstos se añaden otros factores de riesgo, como la pobreza, los estilos de vida (alcoholismo, drogadicción, precariedad, promiscuidad) y nutrición deficiente. Para contraer la TB basta con respirar el aire contaminado. En el caso de la TB-MDR, el riesgo de transmisión es similar. Cuando se deja la TBC-MDR sin control en la comunidad del EP, ésta avanzará a la ciudad y se convertirá en una pesadilla social y en una bomba de tiempo de salud pública.

La estrategia DOTS no ha podido detener el ingreso de una nueva amenaza, surgida en el

\footnotetext{
1 En el caso de Perú, el consumo es generalmente de pasta básica de cocaína y marihuana, a diferencia de los países europeos, donde es común el uso de inyectables intravenosos.

2 Durante los días de visita de mujeres en el EP de Lurigancho, Lima, ingresa un número significativo de trabajadores sexuales y en el interior se adecúa ambientes para su oficio.

${ }^{3}$ De acuerdo al boletín estadístico INPE, marzo 2004, solo 30\% de las personas privadas de libertad de todos los establecimientos penitenciarios del país, había sido sentenciada.
} 
mundo y entre nosotros: es la tuberculosis multidrogo resistente. Las tasas de incidencia de este tipo de TBC están aumentando año a año en nuestro país. El año 1999, se registró una tasa de incidencia en pacientes antes tratados de 12,3\% TBC-MDR. En los últimos siete años, las actividades de control de TB han denotado un empobrecimiento de sus acciones de captación de sintomáticos respiratorios $\left({ }^{4}\right)$. Frente a esta situación, el país está preparando de manera masiva la estrategia DOTS-Plus para el uso de retratamientos individualizados.

Se ha calculado que $43 \%$ de los pacientes con la coinfección VIH-TBC tienen realmente tuberculosis multidrogorresistente, por lo que actualmente se está considerando que en el enfoque de control de la TBC debe intervenir también un enfoque de control de la infección VIH, debido a la vulnerabilidad inmunológica de estos pacientes. La infección VIH en nuestro país todavía es considerada como una epidemia concentrada principalmente en grupos de personas con costumbres sexuales de riesgo. Por lo tanto, los esfuerzos de control de esta enfermedad deben ser concentrados también en estos grupos sociales.

Como vemos, en los lugares donde existe pobreza, conflicto, discriminación y desinterés encontraremos las condiciones adecuadas para una salud amenazada por TBC. La prisión es un ambiente donde se dan estas condiciones, constituyendo esta enfermedad, conjuntamente con las enfermedades mentales, la violencia y el VIH, las principales causas de morbilidad. Estas condiciones de riesgo, no sólo afectan directamente a los presos, sino a todas las personas que están en contacto con las prisiones. Asimismo, las personas luego de permanecer en las prisiones regresan a su quehacer cotidiano, llevando este riesgo de transmisión a la sociedad en su conjunto.

Los datos más recientes del INPE $\left({ }^{9}\right)$ señalan que, en marzo de 2004, existían 30398 personas privadas de su libertad, de las cuales $93 \%$ era del sexo masculino y $7 \%$ del sexo femenino.
Del total de internos en los distintos penales del país, sólo $31 \%$ tenía sentencia; en el resto, su situación jurídica estaba en proceso. El 51\% del total se encuentra en los nueve penales más grandes del país, repartidos como se muestra en la Tabla 4.

Tabla 4.- Personas privadas de su libertad. Perú, 2004.

\begin{tabular}{lr}
\hline Establecimiento penitenciario & PPL \\
\hline Lurigancho, Lima & 7800 \\
Miguel Castro Castro & 1000 \\
Callao, Sarita Colonia & 1183 \\
Socabaya, Arequipa & 607 \\
Trujillo & 1140 \\
Huánuco & 1205 \\
Ica & 545 \\
Picsi, Chiclayo & 900 \\
Castilla, Piura & 1137 \\
\hline
\end{tabular}

Según las normas del INPE, el interno tiene derecho a alcanzar, mantener o recuperar el bienestar físico y mental, proveyéndosele lo necesario para el desarrollo de las acciones de prevención, promoción y recuperación de la salud de la población penal. Dentro de estos lineamientos, ejecuta algunos programas de salud: Programa de control Madre-Niño; Programa de control de Tuberculosis; Programa de control de Enfermedades de Transmisión Sexual y SIDA. En una evaluación realizada en el año 2001, se atendió en los servicios de salud del INPE el $60 \%$ de la población penal. Sin embargo, no existen cifras claras sobre el manejo de los programas que teóricamente son aplicados en los establecimientos penales del país. En informes presentados por la ONG Médicos Sin Fronteras que ha trabajado en el establecimiento más poblado del Perú (Lurigancho), se señala que las condiciones de manejo de estos programas no son los más adecuados; por otro lado, manifiestan que las condiciones de vida de la población penal ponen en alto riesgo de contraer la tuberculosis.

En los EP del Perú, la detección de los casos de TBC se realiza de manera pasiva. Los internos con tuberculosis deben acudir a la clínica del penal 
por tos prolongada, que está asociada a otros signos o síntomas (hemoptisis, fiebre nocturna, baja de peso). La toma de muestra de esputo no reúne muchas veces la garantía que sea realizada en las mejores condiciones técnicas, debido a la falta de información de los consultantes. También, es difícil controlar el reemplazo de muestras negativas de los sospechosos por muestras positivas de los enfermos, en la búsqueda de ganancia secundaria por tener una enfermedad contagiosa. Es muy difícil definir «los contactos» de los pacientes diagnosticados, por el hacinamiento. Adicionalmente, la conducta de los drogadictos con molestias respiratorias no contribuye a la captación de sintomáticos respiratorios. Es difícil realizar exámenes de control de baciloscopia mensuales a los pacientes en tratamiento. En relación al tratamiento, es muy difícil realizar la observación del tratamiento supervisado en boca, por falta de personal, por lo cual algunos establecimientos utilizan un 'colaborador' para que realice esta función. No existen garantías que aseguren que este colaborador realiza adecuadamente su función. Los medicamentos son proporcionados por el Ministerio de Salud. Sin embargo, en algunos establecimientos penitenciarios la comunicación con la Dirección de Salud respectiva no es muy fluida, trayendo como consecuencia que en algunas ocasiones exista desabastecimiento de algunos medicamentos.

En Lurigancho se realizó una evaluación del Programa de Tuberculosis, el año 1998, encontrando que se ha llegado a diagnosticar en promedio 260 casos por año, lo que equivale a una tasa de morbilidad promedio de 3680 casos por 100000 internos; al hacer una comparación de tasas de prevalencía con la población general, da como resultado que existe 30 veces más riesgo de enfermarse que fuera del penal. Sin embargo, estimamos que este número de casos no refleja la situación actual, porque la intensidad de búsqueda de sintomáticos respiratorios no es eficiente por falta de recursos humanos, fiscales y factores conductuales de los administradores de los EP.

\section{IMPLEMENTACIÓN DE UN PROGRAMA DE CONTROL DE TBC EN LOS ESTABLECIMIENTOS PENITENCIARIOS}

Para el año 2002, la incidencia de TB pulmonar en México es de 15,2 por 100000 y se estima 616 por 100000 para la población de prisiones $\left({ }^{10}\right)$. Similar información se logró en Sao Paulo; para el mismo año, se estimó una incidencia de TBC en las prisiones de 707 por 100000 habitantes, mientras que la incidencia en la población general era de 50 por $100000\left({ }^{11}\right)$. En el caso del Perú, se estima que la incidencia de TB en la persona privada de libertad es 25 veces mayor que en la población general.

Frente a esta situación, se plantea una estrategia para la atención integral de la TBC y la coinfección TB-VIH en los penales del país. Para tal efecto, se constituyó un consorcio liderado por la Facultad de Medicina de la Universidad Nacional Mayor de San Marcos, de manera de elaborar un plan en el marco del Proyecto "Fortalecimiento de la prevención y control del SIDA y tuberculosis en el Perú" y lograr la meta "Disminuir de tasa de incidencia de casos positivos de tuberculosis baciloscópica en los nueve penales más grandes del Perú”. El presente plan de acción, tiene como objetivo cubrir las brechas existentes en las estrategias de control de la TB en los penales, mediante siete actividades puntuales: a) Elaboración de una Guía nacional de atención de VIH y TB, TB y TB-MDR en Penales; b) Actualización en estrategia DOTS y capacitación en DOTS-Plus, Coinfección VIH-TB, Control de Infecciones; c) Plan de abogacía para los administradores de EP y autoridades; d) Plan IEC para los internos relacionado a prevención y control de TB; e) Despistaje de VIH en internos; y, f) Diagnosticar y referir internos con VIH/SIDA en los penales a fin de protegerlos adecuadamente de la coinfección TB y de otras infecciones oportunistas.

Al culminar la intervención se espera: a) Aumentar el número de sintomáticos respiratorios examinados de 3413 a 5 000; b) Modificar la 
captación de casos nuevos TB diagnosticados de 360 a 350; c) Aumentar la captación de casos nuevos TB MDR diagnosticados de 20 a 30; d) Aumentar la proporción de internos nuevos que tienen una evaluación específica para TB durante el primer día de ingreso al penal de 0 a $100 \%$; e) Aumentar la proporción de internos que tiene una evaluación específica para TB cada año de $0 \%$ a 50\%; f) Aumentar la proporción de casos TB baciloscópica positiva curados en la estrategia DOTS de $80 \%$ a $90 \%$; g) Aumentar la proporción de casos registrados de TB MDR en tratamiento con DOTS-Plus de $95 \%$ a $100 \%$; h) Disminuir la proporción de abandonos a la estrategia DOTS de 6\% a $3 \%$; e, i) Disminuir la proporción de abandonos a la estrategia DOTS-Plus de $14 \%$ a $10 \%$.

I. Elaboración de una guía nacional de atenciónde VIH y TB, TB y TB MDR en penales. El objetivo general es implementar en los 83 penales del país la referida guía. A nivel nacional se ha diseñado las normas y procedimientos que autorizan las actividades del programa de TB en cada uno de los establecimientos de salud. Sin embargo, estas normas no se han adaptado al trabajo en poblaciones especiales como los penales, por lo que se las considera de necesidad, dadas las características de esta población. Durante los años anteriores, el Programa Nacional de Control de la TBC no ha enfrentado el control de ésta en los penales en forma directa, con una decisión política importante; y sólo ha sido enfocada en forma tangencial. Incluso, esta labor frecuentemente se encuentra bajo el apoyo de la ONG Médicos sin Fronteras y la Cruz Roja Internacional, demostrando la insuficiente importancia por parte del programa en controlar la tuberculosis en los penales.

Lo anterior hace considerar a los penales como un nicho ecológico de gran incremento de la transmisión de la TB, lo cual debe ser enfrentado con estrategias acordes a la realidad penitenciaria y con un apoyo político importante que permitan la viabilización de las estrategias. Es por ello que, la primera actividad a realizarse debe ser la elaboración de una Guía Nacional de atención de VIH y TB (TB y TB-MDR) en Penales, incorporando y adaptando a nuestra realidad las recomendaciones del Comité de la TB en Prisiones (OMS), así como las Guías para la prevención de la TB en los servicios de salud en escenarios de limitados recursos.

II. Actualización en estrategia DOTS y capacitación en DOTS PLUS, coinfección VIH-TB y normas de bioseguridad para TB al personal de salud de las nueve principales prisiones. El objetivo general es fortalecer las competencias de los profesionales de estos penales en el manejo de la estrategia DOTS / DOTS-PLUS, coinfección VIH-TBC y normas de bioseguridad para tuberculosis. Es cierto que la mayor parte del personal está capacitado en las estrategias DOTS y coinfección VIH-TB. Sin embargo, necesita una reactualización de las normas del programa y de nuevas estrategias para el logro de los objetivos. Asimismo, la mayor parte no está capacitado en DOTS-plus (reconocimiento de riesgo de TB-MDR, pruebas de sensibilidad, medicamentos de segunda línea, reacciones adversas, adherencia, entre otros). Por otro lado, existiendo un incremento del número de fracasos a esquemas primarios en esta población, es conveniente capacitarse en el diagnóstico precoz de fracaso a esquemas primarios (esquemas I o II). También, es necesario capacitar en lo referido a la coinfección VIH-TB y su manejo. Por último, la bioseguridad para TB nunca ha sido materia de preocupación en los penales y el personal de salud deberá liderar la mejor implantación de esta estrategia.

La aplicación de la estrategia DOTS y DOTSplus son procesos orientados a lograr mejoras en los programas a través de una detección oportuna de los casos de tuberculosis, un adecuado diagnóstico y tratamiento supervisado. Para esto, debemos contar con recursos profesionales de la salud capacitados en el manejo adecuado de estas estrategias y en normas de bioseguridad. 
Contar con un proceso de capacitación que permita al personal de salud reforzar y actualizar sus conocimientos en el manejo de la estrategia DOTS, DOTS-plus, coinfección VIH-TB y normas de bioseguridad demandará un conjunto de estrategias en las cuales se impulsará la integración enseñanza-servicio; el capacitando reelaborara el conocimiento y consecuentemente modificará su práctica.

III. Plan de abogacía para los administradores de los penales. El objetivo general es implementar, en los nueve principales del país, disposiciones internas que orienten la prevención y control de la TB.

El plan de abogacía consiste en un conjunto de actividades coordinadas para influir en personas, grupos o instituciones con poder de decisión, para promover intereses colectivos, por el bien de la sociedad. La abogacía es un proceso orientado a lograr cambios, reforzar o proponer políticas a través de la comunicación de información, la persuasión y el diálogo. Sus estrategias incluyen principalmente el cabildeo, mercadeo social, información-educación, comunicación. El plan de abogacía debería concretizarse en normas, procedimientos y actitudes de las actividades realizadas. Se espera lograr compromisos que canalicen con el tiempo las mejoras o cambios en leyes, políticas, normas o procedimientos en los EP. Los procesos de abogacía ponen en juego diversos actores sociales y políticos y tienen como objetivo: a) efectuar cambios en una condición social problemática por medio del cambio de una política pública relacionada a ese problema; y, b) reforzar la capacidad de la sociedad civil como actor político.

En la mayoría de penales del Perú no existen normas que fijen acciones para la prevención y control de enfermedades como la TB y el VIHSIDA, debido a: 1) Diversos y graves conflictos que se producen por el hacinamiento de internos; 2) Escasos recursos fiscales con que cuentan los penales; 3) Escasa información y sensibilización de las autoridades ante el problema de TB de los internos; y, 4) Desorganización producida por la intervención de autoridades y personal de diferentes instituciones (INPE, Ministerio de Justicia, Ministerio del Interior, Ministerio de Salud). Por lo tanto, es necesario que se implemente un Plan de Abogacía que influya en las diferentes autoridades de los penales y de los Ministerios relacionados, para que se informen, se sensibilicen, asuman sus responsabilidades, se pongan de acuerdo entre ellos, dirijan acciones de prevención y control de TB en el interior de los penales y se cuente con disposiciones internas que orienten la prevención y control de TB /VIH

IV. Plan IEC relacionado a prevención y control de TB. El objetivo general es elaborar el plan de IEC relacionado a la prevención y control de TB en penales, haciendo uso de las técnicas y medios de esta estrategia.

La comunicación, la educación y la salud coinciden y se encuentran en la persona humana. La comunicación favorece el desarrollo de comportamientos y estilos de vida saludables a través de los medios de comunicación y la comunicación interpersonal, sobre todo en enfermedades que afectan a poblaciones social y económicamente deprimidos. El abordaje de la comunicación y educación son una alianza estratégica para desarrollar prácticas saludables en los internos de los penales, considerando la alta tasa de desconocimiento de los síntomas y del tratamiento de la enfermedad.

El estigma social aún existente sobre la TB pone un peso emocional aplastante en quien lo sufre y su familia, sumándose a ello la falta de información acerca de esta enfermedad, su tratamiento y las medidas preventivas. Estos prejuicios no facilitan el destierro de la enfermedad, y sólo a través de procesos de IEC se favorecerá las mejoras en las condiciones de vida en las zonas de intervención. Esto requerirá la participación social activa y responsable de las personas privadas de libertad, familiar y personal que se relacionen con ellos. Por otro lado, el Taller Internacional de Control de TB en Centros Penitenciarios (San Pedro Sula, 
Honduras, agosto 2003) considera necesario que el Plan de IEC deberá constituir una prioridad para lograr el éxito en la implementación y seguimiento del Plan de Acción y garantizar la participación del personal de salud, del personal de los centros penales, de las personas privadas de libertad, los familiares y la comunidad. Este acuerdo fue ratificado en agosto 2004 en Lima, Perú.

En este contexto, se considera que la experiencia de trabajo y el compromiso de los promotores de salud es un elemento indispensable para la lucha integral contra este problema social, por lo cual se formará promotores de salud en cada uno de los pabellones de los EP. Los promotores conocen su realidad y saben cómo relacionarse con sus familiares y amigos. El autocuidado y la participación activa de los pacientes y sus familiares son elementos claves en la prevención de la TB y la coinfección TB Y VIH. Estos promotores serán capacitados en las señales (síntomas y signos) mas destacados de TBC, para apoyar al personal médico en la detección oportuna de los sintomáticos respiratorios

Como parte de su labor los promotores de salud participaran en: 1) Jornadas de información para las personas privadas de libertad, mediante el desarrollo de cuatro campañas informativas al año en cada uno de pabellones.. Asimismo, nueve campañas masivas en cada penal celebrando el Día Mundial de lucha contra la TB; 2) Ferias educativas e informativas; 3) Un concurso de murales referidos al tema.

Se producirá materiales educativos, teniendo como eje:

1. Brindar información sobre TB TB/MDR y asociación TB VIH/SIDA,

2. Promover los servicios de salud.
3. Apoyar las actividades preventivas promocionales con el equipo multidisciplinario.

4. Abordar los problemas de estigma y discriminación que viven las personas con TB.

5. Ser una persona reconocida que tiene información y apoya a las personas privadas de libertad en el cuidado de su salud.

V. Despistaje de VIH en internos. El objetivo general es el despistaje serológico de infección por VIH en internos de los nueve principales EP del país.

La epidemia del VIH para el año 2000 había sido estimada en 36,1 millones de habitantes afectados a nivel mundial, 1,4 millones para América Latina y para el Perú 76000 personas. En el Perú, hasta el año 2002, se notificó 12 311 casos, estableciéndose una epidemia de tipo concentrada, con $0,26 \%$ en poblaciones de baja prevalencia y $11 \%$ en poblaciones de alta prevalencia (HSH del año 2000), 1,1\% en personas privadas de libertad (centro de reclusión de Lurigancho) $\left({ }^{9}\right)$.

Como población con alta prevalencia de infección por VIH, se ubican los EP y al interior de éstos los denominados "parias" ${ }^{4}\left({ }^{12}\right)$. Las personas privadas de libertad tienen mayor riesgo de contraer el VIH. Se estima que la prevalencia de VIH en esta población es 75 veces mayor que la población general. El $10 \%$ de pacientes diagnosticados de VIH tiene enfermedad por TB en el momento de diagnóstico y entre 20 y $50 \%$ de pacientes con VIH desarrolla TBC en algún momento de sus vidas. Por ello, es fundamental el logro de un adecuado control de la infección por VIH, con la finalidad de disminuir los riesgos, en la medida que los pacientes con VIH son los principales reservorios de TBC (especialmente TB-MDR).

\footnotetext{
4 “parias" son aquellos internos que pasaron el umbral de "lo permitido" y establecieron conductas no aceptadas por la comunidad de internos dentro o fuera del penal. En este grupo se ubican los viciosos, los drogadictos, los violadores; los vínculos de estos internos con los otros son limitados. Portocarrero y col: ob. cit.
} 
No existe una política de despistaje sistemático de VIH en los EP (previa consejería y consentimiento informado). Por consiguiente, se necesita implementar un sistema de investigación del estado serológico VIH de los internos, lo cual permitirá identificarlos oportunamente, un control eficaz de la infección por VIH y disminuir el riesgo de TB y otras infecciones oportunistas mediante el acceso oportuno a la terapia antirretroviral de gran actividad (TARGA). Como resultado, se espera lograr 14808 internos con consejería previa al estudio y con tamizaje serológico para el VIH. El objetivo será un despistaje serológico de infección por VIH en personas privadas de libertad de los nueve principales centros penitenciarios del país.

Se concluye que:

1. Las personas privadas de libertad de los EP tienen tasas de incidencia mayores que la población general en TBC y en VIH.

2. Las condiciones ambientales, culturales y de saneamiento hacen que las personas privadas de libertad de los EP sean consideradas poblaciones de alto riesgo en estas enfermedades.

3. Es necesaria la implantación de estrategias sanitarias diferentes y eficientes para disminuir los riesgos de estas enfermedades.

4. En el último año, la Facultad de Medicina de la Universidad Nacional Mayor de San Marcos está proponiendo mejorar las condiciones de salud de las personas privadas de libertad mediante intervenciones educativas en profesionales de la salud, la formación de promotores y actividades de IEC.

\section{AGRADECIMIENTOS}

Los autores agradecen la colaboración del Dr. José Best y su equipo de salud del Instituto Penitenciario del Perú por la valiosa contribución al presente estudio.

\section{REFERENCIAS BIBLIOGRÁFICAS}

1. Reyes H. El VIH en Establecimientos Penitenciarios. Edición para América Latina. Comité Internacional de la Cruz Roja. Lima: Organización Mundial de la Salud, Oficina Regional para Europa; 2001.

2. WHO. Report 2004. Global tuberculosis control. Surveillance, planning, financing. Geneva: WHO; 2004.

3. Becerra MC, Farmer PE. The problem of drug resistant tuberculosis: an overview. En: Harvard Medical School/ Open Society Institute. Washington: The global impact of drug resistant tuberculosis; 1999.

4. Jave O. El Perú y el control de la tuberculosis [monografía en Internet]. Lima: Pontificia Universidad Católica del Perú [citado el 15 de junio de 2005]. Disponible en: http:// palestra.pucp.edu.pe/index.php?id $=101 \&$ num $=5$

5. Oficina de Estadística, Ministerio de Justicia Instituto Nacional Penitenciario. Estadística población penal Marzo 2004. Lima: Ministerio de Justicia Instituto Nacional Penitenciario; 2004.

6. OPS. El control de la tuberculosis en prisiones. Manual para directores OMS. Comité Internacional de la Cruz Roja. Lima: OPS; 2000.

7. Comisión Nacional Multisectorial en Salud (CONAMUSA). Línea de base de los nueve establecimientos principales penales del Perú. Fortalecimiento de la prevención y control del SIDA y la TB en el Perú. Lima: CARE-Perú; 2004.

8. Alcabes P, Bedell R, Bijl M, Bone A, Curran L, Freudenberg $\mathrm{N}$ y col. El VIH en establecimientos penitenciarios. Edición para América Latina. Comité Internacional de la Cruz Roja. Lima: Organización Mundial de la Salud, Oficina regional para Europa; 2001.

9. Ministerio de Salud del Perú, DGSP ESNP ESNPAC. Guía nacional de Atención de VIH y TB, TB MDR en establecimientos penitenciarios. Lima: Ministerio de Salud del Perú; 2004.

10. Castellanos M. La experiencia de México en el control de la $\mathrm{TB}$ en prisiones. Informe del taller internacional de control de la tuberculosis en centros penitenciarios, Honduras. Tegucigalpa: OPS; 2003.

11. Galesi V. Estimación de la carga del control de la TB en prisiones. Informe del taller internacional de control de la tuberculosis en centros penitenciarios, Honduras. Tegucigalpa: OPS; 2003.

12. Portocarrero J, Cárdenas S, Camacho C, Kcomt C, Gutierrez L, Rojas J. Estudio de percepciones sobre tuberculosis en cuatro penales del Perú. Proyecto Fortalecimiento de la Prevención y control de TB y VIH. Fondo Global. Lima: Consorcio San Marcos; 2004.

Manuscrito recibido el 25 de abril de 2005 y aceptado para publicación el 30 junio de 2005.

Correspondencia: Dr. Eduardo Zarate $C$.

Facultad de Medicina, UNMSM. Av. Grau 750. Lima 1, Perú.

Correo-e:eduzac@ec-red.com 